\title{
A cluster analysis of cyclists in Europe: common patterns, behaviours, and attitudes
}

\author{
Federico Fraboni ${ }^{1}$ (D) Gabriele Prati $^{1}$ (D) Giulia Casu ${ }^{1}$ (D) Marco De Angelis ${ }^{1}$ (D) \\ Luca Pietrantoni ${ }^{1}$ (D)
}

Accepted: 4 March 2021 / Published online: 31 March 2021

(c) The Author(s) 2021

\begin{abstract}
This study uses cluster analysis on a sample of regular cyclists from six European countries (the U.K., the Netherlands, Sweden, Hungary, Italy, and Spain) to shed light on common cycling patterns, demographic characteristics, and attitudes. Participants completed an online survey on cycling behaviour, attitudes towards cycling, discomfort while cycling in mixed traffic, cycling environment and comparative cycling risk perception. A two-step cluster analysis was performed to identify segments of cyclists based on cycling patterns, and a multinomial logistic regression analysis was used to profile the segments. The twostep cluster analysis yielded three components. Leisure-time cyclists cycled almost exclusively for leisure/training, had a clear preference for car use relative to bicycle, and low riding frequency. Resolute Cyclists were characterised by a high variety of cycling trip purpose, a clear preference for bicycle use relative to the car, and high riding frequency. Convenience Cyclists were characterised by cycling for personal business or leisure/training but not for commuting, no evident preference for bicycle vs car, and medium riding frequency. The value of the present study is to highlight commonalities in patterns, characteristics, and attitudes of cyclists in Europe. Our study showed that cycling patterns and habits are linked to psychosocial variables such as attitudes and the cycling environment, explicitly highlighting the importance of discomfort in mixed traffic and the relationship with cycling culture.
\end{abstract}

Keywords Bicyclists - Cluster analysis - Travel behaviour - Mode choices · Attitudes towards cycling $\cdot$ Comparative risk perception $\cdot$ Cycling in mixed traffic

\section{Introduction}

Using the bicycle as a primary or complementary mode of transportation is acknowledged to have multiple benefits both in terms of people's health and wellbeing (Götschi et al. 2016; Kelly et al. 2014) as well as achieving cheaper costs both for the individual

Federico Fraboni

federico.fraboni3@unibo.it

1 Department of Psychology, University of Bologna, Via Filippo Re, 10, Viale Berti Pichat 5, 40126 Bologna, BO, Italy 
and the community and less environmental pollution (de Nazelle et al. 2011; Macmillan et al. 2014). Promoting the use of the bicycle is thus seen as an essential strategy to improve citizens' quality of life and reduce the adverse effects of the car-centric urban mobility plans and policies, which characterised previous decades (Nieuwenhuijsen and Khreis 2016; Mueller et al. 2018).

Various studies focused on identifying cyclists' segments to discern differences in the needs and preferences regarding such mode of transport. More generally, in recent years, studies using attitude-based segmentation to promote environmentally sustainable transport have increased (Haustein and Hunecke 2007). An insightful literature review from Haustein and Hunecke (2007) compares attitudinal, sociodemographic, geographical and behavioural segmentation criteria in multiple studies. Authors concluded that none of the different approaches can claim absolute superiority and that attitudinal approaches show advantages in providing starting-points for interventions to increase cycling use (Haustein and Hunecke 2007). Specifically, clustering cyclists has been proved as an effective strategy to identify meaningful differences in patterns and behaviours (Ahmed et al. 2017; Félix et al. 2017) and develop typologies useful to understand variations and target policies towards the identified groups (Chaloux and El-Geneidy 2019). Félix et al. (2017) also conducted a literature review that appraised cyclists' categorisation methods and compared the obtained cyclists' categories. The authors highlighted some limitations in the current literature: firstly, the studies that have been considered in the review focus on one single city or country, thus not allowing to profile segments in terms of countries and identify meaningful cross-boarder differences. This issue is exacerbated by the fact that studies in different countries use different approaches to segmentation as well (i.e. "Top-Down" approaches such as a priori expert judgment or rule-based classification, versus "Bottom-Up" such as using respondents' data to perform cluster or factorial analysis). Secondly, when profiling segments of cyclists, there is a need to explore motivators, deterrents, and attitudes further. Heinen et al. (2011b) indicate that attitudes toward cycling have a relatively strong impact on the choice to commute by bicycle and suggested that psychosocial factors should receive more attention. Commonalities and interplay between sociodemographic, spatial and attitudinal variables should be further investigated according to Haustein and Hunecke (2007).

Profiling clusters of cyclists has been found to provide deeper insights into their behaviour and characteristics (Ahmed et al. 2017; Kroesen and Handy 2014). Cycling patterns (e.g., motives, frequency) can be considered intrinsic lifestyle choices (Gatersleben and Appleton 2007; Heinen et al. 2011a) and can be influenced by a range of factors including socio-demographics and psychosocial ones. Ahmed et al. (2017) divided their study population into groups according to 'cycling frequency', 'cycling distance' and 'travel planning behaviour' to increase the knowledge of commuters' cycling behaviour. Authors profiled the obtained groups specifically to understand how various factors relate to different types of commuter cyclists' riding decisions. Damant-Sirois et al. (2014) revealed four distinct cyclist types: "dedicated cyclists", "path-using cyclists", "fairweather utilitarians", and "leisure cyclists". The authors discerned which type of cyclists will likely be affected by certain interventions through assessing respondents' answers about motivators, deterrents, and infrastructure preferences. Their work suggests that building a network that is tailored to different cyclist types and emphasising its convenience, flexibility, and speed, can be an effective strategy to increase cycling mode share and frequency among the various groups.

Furthermore, marketing studies show that addressing the average consumer, or in the context of the present study the "average cyclist", is of little use in terms of research impact. Instead, it is more useful to identify different groups of people which 
can be separately addressed because they are motivated by different factors and are affected in different ways by policies and interventions (Anable 2005).

Regarding the European context, previous authors argued that it is crucial to develop targeted efforts to increase shares of sustainable mobility, in particular, cycling (Haustein and Nielsen 2016). It requires an understanding of peoples mobility patterns, motivations and barriers as well as an understanding of differences and similarities between countries. Authors showed that accounting for cross borders differences and similarities allows identifying evidence that could be used to change people's behaviour. Sustainable mobility promotion can find leverages according to similarities or differences in individual countries, with comparable patterns (Haustein and Nielsen 2016). Haustein and Nielsen (2016) focused on identifying mobility patterns clusters across 28 European countries, pinpointing the existence of two clusters for what regards cycling: Practical cyclists and Green cyclists. However, current knowledge on the matter could still be further improved. The present study will thus follow this line of research, comparing people's cycling patterns, attitudes, and psychosocial factors within Europe. Comparisons could empower European policy and decisionmakers with the knowledge to address the diversity of sustainable mobility challenges. We would expect to find commonalities in cycling patterns related to psychosocial factors across countries.

Based on the above considerations, the present study aimed to address the research gap previously highlighted in the literature, explicitly identifying naturally occurring segments in a population of cyclists in six different European countries (U.K., The Netherlands, Sweden, Hungary, Italy, and Spain) basing on cycling patterns and profiling such groups according to demographics and sociopsychological variables. It is possible to identify previous studies that used cluster analysis to highlight natural occurring subgroups in a population of cyclists and to profile segments according to sociodemographic and psychological factors such as attitudes towards cycling (e.g., Nkurunziza et al. 2012; Haustein 2012; Haustein and Nielsen 2016). Very few of them focused exclusively on cycling behaviours and attitudes in Europe. The present study aims to add to this literature branch though providing insights in comparing riding behaviours and attitudes in different European countries. We selected the six countries based on two criteria: cycling mode share and balanced overview of E.U. geographical areas: Northern Europe (U.K. and Sweden); Western Europe (The Netherlands); Eastern Europe (Hungary); Southern Europe (Italy and Spain). According to aggregated data, the E.U. average cycling mode share is $8 \%$ while it substantially differs in each selected country (European Commission 2014). Table 1 shows cycling modal share for each country from the lowest to the highest. The next section will provide a review of the variables that have been studied concerning cycling and have been proved to give valuable insight for developing targeted interventions.

\section{Literature review}

The factors here introduced and reviewed will be used to segment and profile cyclists. The factors are organised into three subsections: Cycling patterns; Cyclists' characteristics; Safety and risk perception. A fourth section discusses cycling culture and its relationship with other variables included in the study. 
Table 1 Cycling Modal Share of the selected countries

\begin{tabular}{ll}
\hline & $\begin{array}{l}\text { Average } \\
\text { Cycling Modal } \\
\text { Share }\end{array}$ \\
\hline U. K & $3 \%$ \\
Spain & $3 \%$ \\
Italy & $6 \%$ \\
Sweden & $17 \%$ \\
Hungary & $22 \%$ \\
The Netherlands & $36 \%$ \\
\hline
\end{tabular}

Percentages of respondents mentioning the bicycle as the mode of transport most often used on a typical day. Data retrieved from Special Eurobarometer 422a "Quality of Transport" http://ec.europa.eu/public_opinion/archives/ebs/ebs_422a_en.pdf

\section{Cycling patterns}

Riding frequency is one of the most considered variables in cycling research. It is possible to find numerous studies in which cyclists have been segmented in groups based on their riding frequency (Bergström and Magnusson 2003; Heinen et al. 2011b; Larsen and El-Geneidy 2011; Winters et al. 2011). It has been shown that subgroups of cyclists, clustered based on their stated riding frequency, differ from one to another (Ahmed et al. 2017; Heinen et al. 2011a). Ahmed et al. (2017) found that adverse weather conditions influenced the decision to whether cycle or not of part-time commuter cyclists and commuter cyclists who do not plan their behaviour. Furthermore, the authors found that long-distance commuters were encouraged by the presence of off-road paths and short and long distance commuters were stimulated to ride to work when the weather was favourable. Similarly, Heinen et al. (2011a) found that two groups of part-time cyclists exist: occasional cyclists and frequent cyclists. Occasional cyclists were more influenced by favourable weather conditions. In contrast, frequent cyclists were discouraged from cycling by more practical barriers such as wind speed and the need to be at multiple locations. Segmenting cyclists based on riding frequency aided identifying differences in mode choice between seasons (Bergström and Magnusson 2003) and influenced the likelihood to ride (Winters et al. 2011).

Cycling trip purpose is another factor that has been used frequently to identify different typologies of cyclists. In a study by Streit et al. (2014) trip diaries were used to segment cyclists by riding frequency and why they use the bicycle (leisure vs commuting). A similar approach can be found in other studies (Heinen et al. 2011a; Dill and Voros 2007). Significant differences between groups were found when using cycling purposes (commuting versus non-commuting) as segmentation criteria (Kroesen and Handy 2014; Rondinella 2015). Segmenting cyclists based on cycling trip purpose helped to shed light on habits and patterns of cyclists and to understand that recreation or sport seems more relevant than commuting as cycling trip purpose (Burbidge and Goulias 2009; Moudon et al. 2005; Sener et al. 2009).

Modal split is often considered in research when it comes to assessing mobility patterns and changes over time or the effectiveness of interventions in promoting a modal shift towards active mobility (Barberan and Monzon 2016; Rayaprolu et al. 2018; Song et al. 2017). It has proven to be a relevant factor for identifying bicycle use habits. For example, Barberan and Monzon (2016) found that people prefer to drive their cars to commute to 
work and almost equally to walk and cycle to their place of study. Jensen (1999) categorised cyclists according to their preferences about using public transport or the bicycle. He distinguished three categories: the "cyclists of heart" which cycle for the enjoyment of the experience and decide not to own a car; the "cyclists of convenience" which cycle because it is the most convenient mode; the "cyclists of necessity" which cycle because they cannot afford a car. These categories are useful to structure mode selection as a function of choice, whether from principle or utility or mode captivity.

\section{Cyclists' characteristics}

Sociodemographic characteristics of cyclists have been investigated in a variety of studies. Gender differences in cycling have been identified in previous studies (e.g. Garrard et al. 2012; Heesch et al. 2012; Wittmann et al. 2015). Prati et al. (2019) assessed gender differences in cycling patterns and attitudes towards cycling in a European sample, finding small but significant differences in terms of perceptions, attitudes, and behaviours. Other studies showed gender influencing the use of the bicycle as a means of transport (Bergström and Magnusson 2003; Heinen et al. 2010; Prati 2018) and the reason to use the bicycle. Women tend to cycle mostly for non-commuting trips such as travel with children and to carry shopping and other goods (Pucher and Buehler 2012). The country of residence is also an essential factor when looking at cycling patterns. There are substantial historical differences in the share of cycling between the countries considered in the present study, in particular, The Netherlands, Sweden and Hungary cycling share differ substantially from Italy, Spain and U.K, according to latest aggregated data (European Commission 2014). For instance, in Italy, about $6 \%$ of respondents mentioned the bicycle as the mode of transport most often used, while in the Netherlands this percentage rises to $36 \%$. It is essential to point out that the Netherlands can be considered an outlier regarding bicycle shares.

Attitudes toward cycling have been investigated in previous studies and have been found to influence bicycle use, mode choice, and purpose for cycling significantly (Gatersleben and Appleton 2007; Gatersleben and Uzzell 2007; Heinen et al. 2011b). To our knowledge, this is the first study that attempts to profile cyclists in terms of attitudes with a multi-country European sample. Heinen et al. (2011b) reported that when commuters find cycling to be comfortable, flexible, and time-saving, they were more prone to cycle to work as well as individuals who consider cycling environmentally friendly, healthy and mentally relaxing (Heinen et al. 2011b). It has been argued that the combination of motivational and attitudinal factors affecting travel choice for distinct groups of people is often neglected when it comes to cycling research. Other authors, for example, provided evidence that although land use characteristics have some significance in explaining travel behaviour, individual attitudes are often more strongly associated with travel behaviour than land-use policies which promote higher densities (Handy et al. 2005; Mercado and Páez 2009; Susilo and Maat 2007; Susilo and Waygood 2012). Authors have argued that land-use policies promoting mixed-use and higher density developments may not have a material effect on travel behaviour unless individuals' attitudes are also changed (Bagley and Mokhtarian 2002; Susilo et al. 2010). Further studies additionally highlighted the importance of assessing how cycling is socially viewed, considering the values associated to cycling, the image, and the aspects perceived as requirements for being able to cycle appropriately (Aldred and Jungnickel 2014). Attitudes towards cycling may be related to many different attributes, such as fun, relax, comfort, convenience, accident risk, environmental benefits, health, flexibility (Barberan and Monzon 2016). Those attributes have been grouped by authors trying 
to find the underlying latent variables that could measure such attitudes (Fernandez-Heredia et al. 2016; Muñoz et al. 2016). Anable and Gatersleben (2005) addressed the evaluations that the person holds regarding cycling as a transportation mode and how they impact on mobility choices. In their work, attitudes were grouped into two categories: hedonic and instrumental attitudes. Hedonic attitudes, initially addressed as affective, refer to the positive emotions experienced while cycling such as excitement, pleasure, and control. Most of the studies examining the affective experience of travel focused on the negative experience of stress, in particular for commuters (Koslowsky 1997; Gulian et al. 1990). In the present study, we focused on positive emotions such as considering cycling as mentally and physically relaxing and pleasant. Instrumental attitudes refer instead to the utility or functionality of cycling (e. g. predictability, safety, and convenience). In literature, such factors have been investigated to understand their impact on people's mobility choices (Bamberg and Schmidt 2001; Van Lange et al. 1998). Given the increased public awareness of the benefits of cycling, they have been considered in the present work as well, mainly comprising attitudes towards the benefits of cycling such as personal health (Woodcock et al. 2014; Useche et al. 2019), environmental benefits (Karanikola et al. 2018; Pucher and Buhehler, 2017), low-cost (de Nazelle et al., 2011; Macmillan et al., 2014) and flexibility of cycling. Stinson and Bhat (2004) showed that cyclists who considered cycling as "flexible" and "with predictable travel time" were more likely to use it as a mode of transportation, in particular for commuting to work. Heinen et al. (2011b), using factor analysis on a bicycle attitudinal characteristics scale, found support for an "awareness" dimension including the perception of health, environmental and economic benefits of cycling.

\section{Safety and risk perception}

An important aspect connected to cycling in mixed traffic is the provision and quality of cycling infrastructure. How they are perceived may have an impact on whether a cyclist feels safe or at risk and has often been considered in the literature. Manton et al. (2016) presented a broad overview of critical infrastructural and traffic factors affecting cyclists' risk perception. These include the presence of cycling facilities, driving lane width, and pavement surface (Bill et al. 2015; Lawson et al. 2013).

When it comes to promoting cycling and identifying measures to make cycling safer, an interesting line of research has focused on the impact of the perceived discomfort while cycling on roads with mixed traffic and its relationship with perceived safety. It has been shown that cyclists perceived a higher feeling of safety on roads with physical separation from motorised vehicle traffic while perceiving to be most at risk on roads with mixed traffic (Heinen et al. 2010; Jensen et al. 2007). Major streets with shared lanes are associated with greater perceived risk (Winters et al. 2011). Félix et al. (2019) identified the feeling of discomfort and fear of cycling with motorised traffic as the first deterrent for non-cyclists and second deterrent for cyclists.

Perceived risk associated with cycling is an important topic in literature, as cycling is often associated with safety concerns (Heinen et al. 2010; Schepers et al. 2014; Wegman et al. 2012). Indeed, bicyclists are considered to be vulnerable road users (VRUs; e.g., Manton et al. 2016; Siman-Tov et al. 2012) due to lower mass, stability and lack of physical protection (Schepers et al. 2014). Thus, cycling is objectively associated with a higher risk of injury than travelling by car or other means of transportation (Heinen and Handy 2012; Wegman et al. 2012). High-risk perception and safety concerns are considered among the most significant psychosocial barriers that prevent people from 
cycling more (Koglin and Rye 2014; Pucher and Dijkstra 2000; Majumdar et al. 2020). Cyclists' perception of risk regarding safety outcomes is deemed to shape mode choice (Puchades et al. 2018; Ul-Abdin et al. 2019). Specifically, the risk of having a crash is found to be one of the most frequently mentioned deterrents to cycling (Parkin et al. 2007; Heinen et al. 2010), including among those who cycle regularly (Bauman et al. 2008). Authors have shown that when people are asked to rate their chances of experiencing certain illnesses, accidents, and other problems, it is essential to address the unrealistic optimism bias (Friedman 2011) thus assessing risk perception in comparison to an average peer of the same age and gender. The unrealistic optimism bias is the tendency of people of overestimating the risk of others and underestimating the same risk for oneself (Weinsten, 1980). When assessing risk perception, other studies (Martha and Delhomme 2009; Schwarzer 1999) suggested highlighting the difference between the perceived risk for oneself (personal risk perception) and the perceived risk for others (general risk perception). Therefore, studying people's subjective opinions regarding safety and comparative risk is remarkably relevant for increasing the use of the bicycle, especially because perceived risk can cause cyclists to shift to other modes or even avoid trips (Heinen et al. 2010). On a practical level, a better understanding of risk perceptions of different groups of cyclists could help to tailor solutions to increase bicycle usage for daily trips.

Lastly, another dimension investigated in the cycling field is motorised vehicles/ truck drivers' behaviour and its evaluation from the cyclists' point of view. From a psychosocial perspective, the literature showed that issues arise in bicycle-motorised vehicles interactions. Mainly, cyclists frequently report being abused and harassed by drivers (Heesch et al. 2012; O'Connor and Brown 2010). Other studies showed that cyclists' presence on the road is often considered annoying by both professional and domestic drivers (Basford et al. 2002). Furthermore, it is particularly interesting to note that cyclists' risk perception and safety concerns were found to be intensified by their ratings of motorists' aggressive driving (Bauman et al. 2008). The perception that motorists do not respect cyclists, as well as cyclists' concerns of motorists' behaviour, was reported to be a relevant deterrent to cycling (Félix et al. 2019). Authors argued that defining cyclists as vulnerable road users do not capture the social and cultural aspects that characterise the membership within the group, thus, proposed that cyclists should be considered minority road users instead (Prati et al. 2017). The conceptualisation of minority (Seyranian et al. 2008) implies that individuals could be the target of specific negative behaviours by majority individuals. Cyclists frequently report aggression and harassments by motorised road users (Aldred and Crosweller 2015; Fruhen and Flin 2015; Johnson et al. 2014). Thus, we would expect that cyclists from countries with low-cycling culture would evaluate motorists' behaviour worse than cyclists from countries with strong cycling culture. Furthermore, a mixed-method study showed that serious leisure cyclists frequently experience intimidation and harassment by motorised vehicle drivers (O'Connor and Brown 2010). To our knowledge, no studies focussed on assessing differences in the evaluation of motorists' behaviours between multiple segments of cyclists. In the present study, we will explore the relationship between the evaluation of motorists' behaviours and belonging to a specific segment of cyclists, expecting to find more negative assessment of motorists behaviour in the group of participants using the bicycle for leisure or sports activities. 


\section{Cycling culture}

Mobility culture is an often mentioned and investigated concept in relation to bicycle use (e.g., Aldred and Jungnickel 2014; Haustein et al. 2020; Haustein and Nielsem 2016; Oosterhuis 2016). According to Haustein et al. (2020) mobility culture should be intended as composed by both tangible (e.g., the built environment) and intangible (e.g., norms and attitudes towards transport mode) factors. Both categories influence people's knowledge and beliefs about transport as well as everyday mobility patterns. Cycling culture should thus be considered a product of the official planning and policy work in a city and the built environment, including the cycling infrastructure, urban density, and how people perceive and experience cycling as a means of transport (Haustein et al. 2020). Cities and, more in general countries, are often addressed as having a strong cycling culture (Pucher and Buehler 2017) when they present higher levels of provision and quality of cycling infrastructure and high shares of bicycle use as an everyday means of transport. For instance, in countries like the Netherlands and Sweden, cycling is already a substantial part of daily mobility in several cities (Pucher and Buehler 2008; Haustein et al. 2020) and are generally considered as strong cycling cultures. On the other hand, countries such as Italy, Spain and the U.K are considered as having low-cycling or emerging cycling cultures (Aldred and Jungnickel 2014; Oosterhuis 2016). Haustein et al. (2020) showed that differences in cycling levels in two Northern-European capital cities are related to differences in the citizens' perceptions and experiences, reflecting differences in transport policies and planning initiatives. The present study considers people's perceptions of the cycling environment and attitudes towards cycling to investigate possible commonalities or differences in countries with diverse cycling cultures.

\section{Materials and methods}

\section{Procedure}

An online survey was administered between 27th January and 5th February 2018, to a panel of respondents in six countries (Hungary, Italy, Spain, Sweden, The Netherlands, United Kingdom) who had previously agreed to take part in data collection. The online panel was bought from Qa survey company. Survey companies hold a significant amount of demographic data on their panellists and keep them up to date. It allows targeting surveys at specific groups and obtaining a particular sample, such as a nationally representative sample or a female-only sample. The sample within each country was required to meet the following criteria: All respondents must make at least one cycle trip per month (on average); At least $50 \%$ of respondents must be regular cyclists (i.e. make on average $>2$ cycle trips per week); At least $30 \%$ of the sample must be female; At least $10 \%$ of the sample must be aged 50 years or more. The inclusion of participants aged 50 or more years old was particularly relevant to assess whether there were significant differences between young and elderly cyclists. The average completion time was around $30 \mathrm{~min}$. A pilot version of the questionnaire was administered to 60 participants, 30 in The Netherlands, and 30 in the United Kingdom. After examining the pilot questionnaire data, the survey was updated with the new wording of items that produced anomalous replies. Pilot survey data was not included in the final dataset due to changes in the post-pilot questionnaire. Then, 
the questionnaire's finalised version was translated, uploaded to a custom online survey platform, and administered to participants.

\section{Data and participants}

A total of 2389 participants completed the questionnaire. Of these, 1171 (49.1\%) were male, $1210(50.6 \%)$ were female and $8(0.3 \%)$ identified themselves as transgender. Given that the sample of transgender participants was too small to be comparable with the other two categories, it was not included in the subsequent analyses, leaving a sample of 2,381 cyclists.

\section{Measures}

The questionnaire included the following sections: Cycling frequency, Trip purpose and Modal split between bicycle and car which were used as segmentation variables; Sociodemographic characteristics, Attitudes towards cycling, Infrastructure rating, discomfort while cycling in mixed traffic, Motorists' behaviour rating and Comparative cycling risk perception which were used as profiling variables.

Cycling frequency has been considered as the product of the number of days cycled per month and months cycled per year. It was measured by the product of 2 items: "How many months a year do you normally cycle?" with answers from 1 to 12 , and "In general, during these months, how often do you cycle?" with answers on a 5 -point scale $(1=$ daily, $2=3$ or more days per week, $3=1-2$ days per week, 1 to 3 times a month, $5=$ less than once a month).

Trip purpose. Respondents were asked why they were making their cycle journeys using the following three dichotomous (Yes/No) items: commuting/travelling to or from work/ college, personal business (e.g., shopping/entertainment, health appointments, or visiting family/friends), and leisure/training. Non-commuting trips have been divided into two categories that are leisure and sports trips (such as bike tour, sightseeing bike ride, competitive cycling training, or bike race) and personal business trips (such as using the bike for shopping, visiting relatives or friends, health appointments). Cycling trip purpose has been used, in the present study, to discern differences and similarities among European cyclists and it is particularly relevant since it can help to identify strategies to increase cycling levels between different segments of the population. In the present study, cyclists have been clustered according to the reason why they cycle, explicitly aiming to identify differences between commuting (i.e., travelling to or from work/college) and non-commuting (i.e., personal business and leisure/training) trips.

Modal split between bicycle and car was assessed using two questions about the car and bicycle usage during months of cycling (i.e., "In general, during the months you cycle, how often do you travel in a car, whether as a driver or passenger?") which were both measured on a 6-point scale ( $1=$ daily, $2=3$ or more days per week, $3=1-2$ days per week, 1 to 3 times a month, $5=$ less than once a month, $6=$ never). To investigate respondents' use of car or bicycle as the primary means of transport, answers to these items were combined into a new modal split variable coded as $1=$ more car than the bicycle, $2=$ same, $3=$ more bicycle than the car, and $4=$ bicycle only. Clustering cyclists concerning modal split brought insights on their habits and helped understand if the increase in cycling demand could grow (Streit et al. 2014). It becomes particularly relevant to the present 
study because it can allow discerning which group has higher potential to increase the use of sustainable mobility.

Cycling environment was assessed through a single item: "What type of environment do you make the majority of your cycle trips within?" with four response options (i.e., city, town, village, rural).

Sociodemographic characteristics. The sociodemographic form asked for age, gender, country of residence, and job status. As for the latter, participants were asked to select one of the following options: full time employed, part-time employed, self-employed, not currently working, students, homemaker, full-time caregiver and retired. Responses were recategorised into three groups, those who are actively involved in a job (employed), those who are studying and those who have no contractual employment or have retired (unemployed). As advocated by Félix et al. (2017), the present study aimed at assessing clusters of cyclists at a European level, exploring differences and similarities in terms of sociodemographic characteristics of different type of cyclists in different countries.

Attitudes towards cycling. To assess hedonic attitudes towards cycling, we used three items that expressed positive feelings towards cycling. Instrumental attitudes were measured through four items measuring the functionality and convenience of the bicycle. Finally, four items were used to evaluate the expected benefits of cycling. We added flexibility (Stinson \& Bhat, 2004) as an additional benefit to health and environmental benefits. All items were rated on a 5-point scale ( $1=$ completely disagree to $5=$ completely agree). Exploratory factor analyses with principal axis factoring yielded a one-factor solution for all three attitudes towards cycling, with no differences across countries. Cronbach's alpha reliability coefficient was 0.85 for hedonic attitudes, 0.83 for instrumental attitudes, and 0.75 for benefits. Items are listed in Table 2 .

Infrastructure rating. We asked the participants to evaluate on a 5-point scale $(1=$ very poor to $5=$ excellent) the overall level of the cycling infrastructure with two items referring to the quality and provision of the infrastructure (i.e., "How would you rate the cycling

Table 2 Items measuring Attitudes Towards Cycling

\begin{tabular}{lc}
\hline How far do you agree that you cycle because... & Items \\
Hedonic Attitudes & it is pleasant? \\
it is mentally relaxing? & it is physically relaxing? \\
Instrumental Attitudes & it is comfortable? \\
of traffic safety? & of personal security? \\
it is time-saving? & Benefits \\
it has benefits for the environ- & ment? \\
it has benefits for personal & health? \\
it has economic benefits? & it is flexible?
\end{tabular}


infrastructure in terms of the level of provision of cycling infrastructure?"; How would you rate the cycling infrastructure in terms of quality of the infrastructure?"). The two questions were introduced by the sentence "Thinking about the environment you mainly cycle in...". Cronbach's alpha reliability coefficient was 0.92 . In the present study, respondents' evaluation of cycling infrastructure in terms of quality and provision has been included to understand whether it would increase the chances of belonging to one cluster compared to the others.

Discomfort while cycling in mixed traffic. Participants were asked to assess their level of discomfort when cycling in the following six scenarios: (1) a path separated from the street, (2) a two-lane (one in each direction) residential commercial shopping street, and no bike lane, (3) the previous scenario with a striped bicycle lane added, (4) a major urban or suburban street with four lanes (2 each direction) and no bike lane, (5) the previous scenario with a striped bike lane added or (6) with a bike lane separated from traffic by parked cars or a kerb. All items were rated on a 5 -point scale $(1=$ very comfortable to $5=$ very uncomfortable). Exploratory factor analyses with principal axis factoring and quartimax rotation yielded a two-factor solution. The first factor was composed by items assessing the feeling of discomfort in mixed traffic with no cycling infrastructure (items 2, 4; with a factor loading of 0.91 and 0.74 respectively) while the second factor included items investigating the feeling of discomfort while riding on streets with cycling infrastructure (items 1, 3, $5,6)$. Since factor 2 assessed the feeling of discomfort when using cycling infrastructure, providing some protection from motorised traffic, we focused on factor 1 to assess the discomfort of cycling in mixed traffic. Cronbach's alpha reliability coefficient was 0.81 .

Motorists' behaviour rating. To explore in which way cyclists perceive and evaluate the behaviour of motorists, the participants replied to the following question using a 5-point scale $(1=$ very poor to $5=$ excellent): "How would you rate the driving behaviour of motorists and van/truck drivers within the environment you mainly cycle in?".

Comparative cycling risk perception. As done in previous studies (Friedman 2011; Martha and Delhomme 2009; Schwarzer 1999), we assessed the comparative perception of risk while cycling by asking participants to estimate their likelihood of being involved in an accident compared to that of their counterparts (i.e. "Compared to other bicycle riders of my age and sex, my risk of being involved in a traffic accident is?). The item was rated on a 5 -point scale $(1=$ much smaller to $5=$ much higher $)$.

\section{Statistical analysis}

Data were analysed using IBM SPSS version 23. Differences in respondents' characteristics between countries were examined using analysis of variance (ANOVA) with Welch's correction and Games-Howell pairwise comparisons for age, and $\chi^{2}$ tests with posthoc $\mathrm{z}$-scores and Bonferroni correction for gender, employment status, bicycle use and evaluation of motorists' behaviour.

To identify segments of cyclists based on cycling patterns a two-step cluster analysis was performed using both categorical (i.e., cycling trip purpose, and relative use of the car vs bicycle) and continuous (i.e., riding frequency) segmentation variables. Cyclists were clustered according to cycling patterns for two main reasons: first because, following the literature review, it has been proven to be a meaningful way to cluster cyclists and, secondly, to allow comparing cycling behaviours and attitudes in different European countries, addressing research gaps identified by Félix et al. (2017). To our knowledge, it has never been done with a sample from six different countries. Secondly, using many 
variables in the segmentation part would have led to overly complex segments, leading to difficulties in discussing results in a meaningful way. We thus decided to use cycling habits (i.e., Cycling frequency, Trip purpose and Modal split) to identify the segments as those variables reflected the actual usage of the bicycle by participants and could allow defining meaningful segments which would later be profiled in terms of sociodemographic and psychosocial characteristics. Two-step cluster analysis first pre-clusters cases into many small sub-clusters, using a sequential clustering algorithm. Nearby sub-clusters are then recursively merged to form the final cluster solution, using an agglomerative hierarchical clustering algorithm. The SPSS two-step clustering component requires only one data pass in the procedure. Automatic selection of the optimal number of clusters was based on the log-likelihood as the distance measure between clusters and Schwarz's Bayesian information criterion (BIC). The silhouette coefficient, which compares the average withincluster cohesion with the average between-cluster separation, was examined to assess the goodness-of-fit of the cluster solution. Values between 0.20 and 0.50 indicate a fair fit, and values of 0.50 or more a good fit (Sarstedt and Mooi 2014). For validation and interpretation of the cluster solution, $\chi^{2}$ tests and ANOVAs were performed on categorical and continuous segmentation variables, respectively. Further between-segment differences were examined with ANOVA for age, attitudes towards cycling, cycling environment, discomfort while cycling in mixed traffic, cycling infrastructure, motorists' behaviour ratings and cycling risk perception, and with $\chi^{2}$ tests for the country, gender, employment status and cycling environment.

Variables listed in the previous paragraph were then entered as independent variables in a multinomial logistic regression analysis aimed to profile the segments. Segment membership was set as the outcome variable. The model $\chi^{2}$, Pearson and deviance tests, and Nagelkerke Pseudo- $\mathrm{R}^{2}$ were considered to assess the goodness-of-fit of the logistic regression model. A significant model $\chi^{2}$ indicates that the model with its independent variables fits the data better than a model without those variables; nonsignificant Pearson and deviance tests indicate nonsignificant differences between the observed and the predicted probabilities; and Nagelkerke R2 values larger than 0.20 represent an acceptable approximate amount of explained variability (Hosmer and Lemeshow 2000). Odds ratios and 95\% confidence intervals (C.I.s) were reported for each independent variable.

Interpretation of results was based on both statistical significance $(p<0.05)$ and measures of effect size: Cramer's V of 0.10 was considered small, 0.30 medium, and 0.50 large; $\eta 2$ of 0.01 was considered small, 0.06 medium and 0.14 large; Cohen's d of 0.20 was considered small, 0.50 medium, and 0.80 large; and O.R.s of 1.5 were considered small, 3.5 medium, and 9 large (Cohen 1988).

\section{Results}

\section{Respondents' characteristics}

The total sample $(\mathrm{N}=2,381)$ was $50.6 \%$ female and mean aged 43 years $(\mathrm{SD}=14.34)$, with $67.8 \%$ being employed. Concerning the bicycle use, $365(15.3 \%)$ participants used a bicycle 1-3 times a month, other 707 (29.7\%) participants cycled 1-2 days a week, 872 (36.6\%) did so three or more days a week, and the remaining 437 (18.4\%) cycled daily. The cyclists' characteristics in the total sample and by country are displayed in Table 3. Participants are approximately equally distributed across the selected countries. Cyclists 


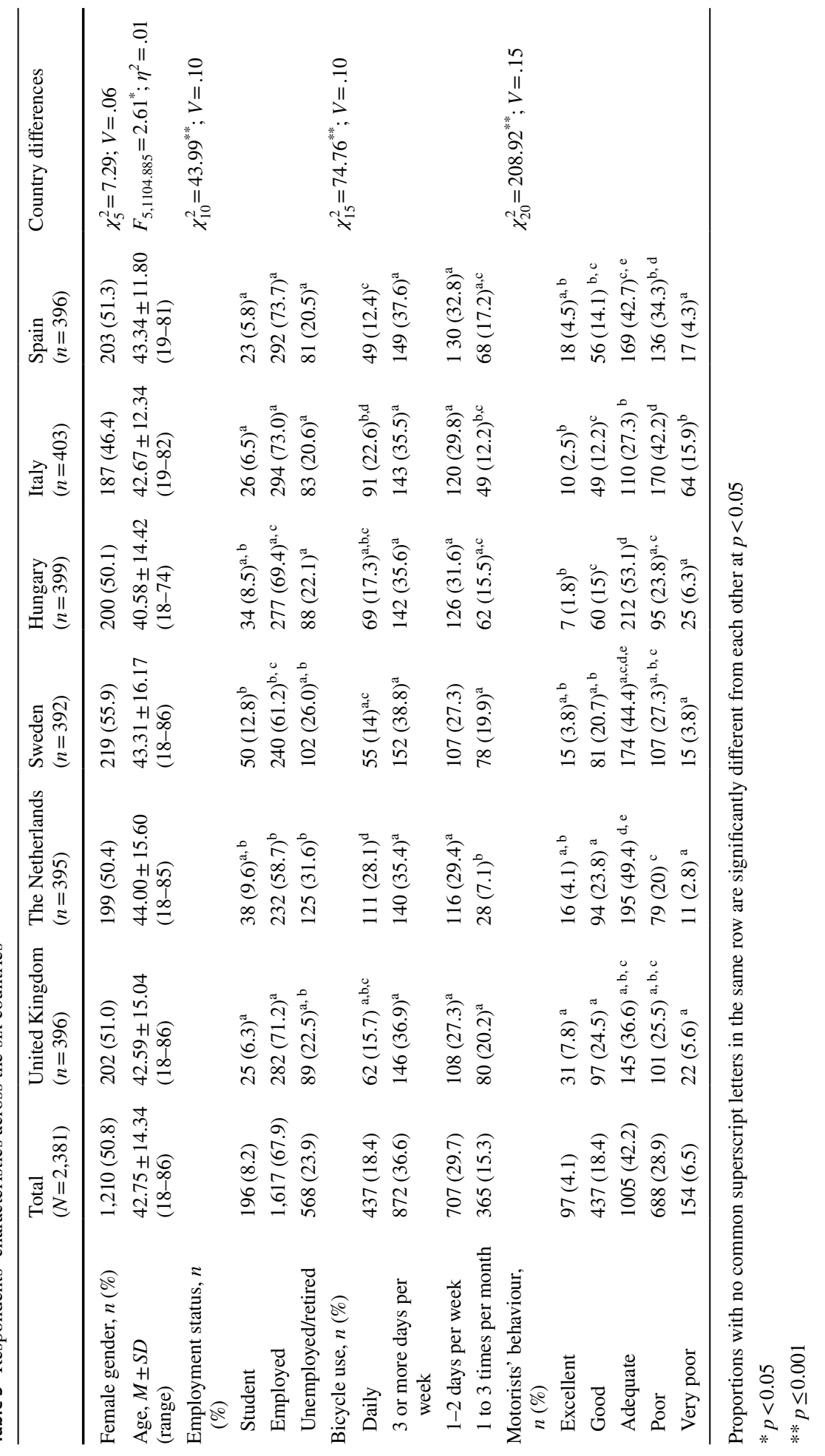


from Hungary were slightly younger than those from The Netherlands $(\mathrm{d}=0.23)$ and Spain $(d=0.22)$. Chi-square tests followed by pairwise comparisons indicated that there were more cyclists who were students in Sweden compared to the United Kingdom, Italy, and Spain, more cyclists who were employed in the United Kingdom, Italy, and Spain than in The Netherlands and Sweden, and more cyclists who were unemployed/retired in The Netherlands than in Hungary, Italy, and Spain. Regarding bicycle use, participants from The Netherlands cycled daily significantly more than participants from the U.K., Sweden, Hungary and Spain. Cyclists using the bicycle 1 to 3 times per month were more in the U.K and Sweden than the Netherlands and Italy, as well as in Hungary compared to the Netherlands.

Regarding motorists' behaviour, cyclists from the U.K. rated it as excellent significantly more than cyclists from Hungary and Italy. Likewise, cyclists from Hungary and Italy rated motorists' behaviour as good significantly less than cyclists from the U.K, the Netherlands and Sweden. Also, participants from Spain rated motorist's behaviour as good significantly less than participants from the U.K. and the Netherlands. Furthermore, cyclists from Italy rated motorist's behaviour as poor significantly more than participants from all other countries.

\section{Segments of cyclists based on cycling patterns}

In two-step cluster analysis, the best solution following automatic clustering based on BIC and log-likelihood distance measures included three segments (Table 4). The average Silhouette coefficient was 0.40 , indicating fair-to-good cohesion and separation. The ratio between the largest and smallest cluster was 1.15 , indicating balanced cluster sizes.

Table 4 Segments of cyclists on the segmentation variables

\begin{tabular}{lllll}
\hline & $\begin{array}{l}\text { Leisure-time } \\
\text { Cyclists } \\
(n=727)\end{array}$ & $\begin{array}{l}\text { Resolute } \\
\text { Cyclists } \\
(n=839)\end{array}$ & $\begin{array}{l}\text { Conveni- } \\
\text { ence Cyclists } \\
(n=815)\end{array}$ & Segment differences \\
\hline $\begin{array}{l}\text { Trip purpose } \\
\text { Commuting }\end{array}$ & $0 \%^{\mathrm{a}}$ & $89.5 \%^{\mathrm{b}}$ & $0 \%^{\mathrm{a}}$ & $\chi_{2}^{2}=2016.20^{*} ; V=.92$ \\
Personal business & $0 \%^{\mathrm{a}}$ & $64.6 \%^{\mathrm{b}}$ & $100 \%^{\mathrm{c}}$ & $\chi_{2}^{2}=1598.23^{*} ; V=.82$ \\
Leisure/Training & $100 \%^{\mathrm{a}}$ & $50.5 \%^{\mathrm{b}}$ & $61.8 \%^{\mathrm{c}}$ & $\chi_{2}^{2}=484.01^{*} ; V=.45$ \\
Modal Split & $63.8 \%^{\mathrm{a}}$ & $14.8 \%^{\mathrm{b}}$ & $41.5 \%^{\mathrm{c}}$ & $\chi_{6}^{2}=490.36^{*} ; V=.32$ \\
More car than bicycle & $17.3 \%^{\mathrm{a}}$ & $26.0 \%^{\mathrm{b}}$ & $27.7 \%^{\mathrm{b}}$ & \\
Same & $16.1 \%^{\mathrm{a}}$ & $50.1 \%^{\mathrm{b}}$ & $30.8 \%^{\mathrm{c}}$ & \\
More bicycle than car & $9.2 \% \%^{\mathrm{b}}$ & $0 \%^{\mathrm{c}}$ & \\
Bicycle only & $2.8 \%^{\mathrm{a}}$ & $173.44^{\mathrm{b}}$ & $106.73^{\mathrm{c}}$ & $F_{2,1544.35}=313.33^{*} ; \eta^{2}=.19$ \\
\hline Cycling frequency & $55.69^{\mathrm{a}}$ & & & \\
\hline
\end{tabular}

Values with no common superscript letters in the same row are significantly different from each other at $p<0.05$

† Proportions of cyclists who responded "Yes"

$\doteqdot$ Riding frequency was computed as the number of months per year in which the person usually cycles and the number of days spent cycling each month. Range $2-365(M=114.65, S D=110.10)$

${ }^{*} p \leq 0.001$ 
The first segment $(n=727)$ constituted $30.5 \%$ of cyclists and was characterised by cycling exclusively for leisure/training, a clear preference for car relative to bicycle, and low riding frequency. This segment was named Leisure-time Cyclists. The second segment $(n=839)$ constituted $35.2 \%$ of cyclists and was characterised by a high variety of cycling trip purpose, a clear preference for bicycle relative to the car, and high riding frequency. This segment was called Resolute Cyclists. The third segment $(\mathrm{n}=815)$, which corresponded to $34.2 \%$ of respondents, was characterised by cycling for personal business or leisure/training but not for commuting, no evident preference for bicycle vs car, and medium riding frequency. This segment was identified as Convenience Cyclists. All comparisons between segments on the segmentation variables were significant at $p<0.001$, with medium to large effect sizes (Table 5).

In comparing the three segments, additional variables were added to provide a broader view of the distinctive characteristics of each group of cyclists (Table 5). Specifically, it was sought to understand whether there were significant differences between the groups in terms of attitudes towards cycling, cycling environment, the degree of discomfort while cycling in mixed traffic, the evaluation cyclists would express about the quality and provision of infrastructure, a general assessment of motorised vehicles drivers' behaviour on the road and the subjective perception of risk associated with cycling. As for the cycling environment, $\chi^{2}$ test indicated that both Resolute Cyclists and Convenience Cyclists cycled in a city or town environment significantly more, and cycled in a rural setting significantly less, compared to Leisure-time Cyclists. Convenience Cyclists made the majority of their cycle trips within a village environment considerably more than both Resolute Cyclists and Leisure-time Cyclists. One-way ANOVA revealed that participants that belong to the Resolute Cyclists cluster expressed slightly less feeling discomfort while pedalling in mixed traffic, which is characterised by the absence of a dedicated bike path, than the groups of Leisure-time Cyclists $(\mathrm{d}=0.20)$ and Convenience Cyclists $(\mathrm{d}=0.15)$. Moreover, Resolute Cyclists rated the quality and provision of the cycling infrastructure and the motorists' road behaviour significantly higher than Leisure-time Cyclists $(\mathrm{d}=0.47$ and 0.21 , respectively) and Convenience Cyclists $(\mathrm{d}=0.16$ and 0.13 , respectively), with small-to-medium effect sizes. Finally, Resolute Cyclists and Convenience Cyclists reported a slightly lower cycling risk perception than cyclists of the Leisure-time Cyclists category $(\mathrm{d}=0.16$ and 0.15 , respectively).

\section{Profiling of the segments}

In the multinomial logistic regression analysis, the outcome variable was segment membership, coded as 1=Leisure-time Cyclists, $2=$ Resolute cyclists, and $3=$ Convenience Cyclists. The Leisure-time Cyclists' segment was used as the reference category. Results are displayed in Table 6.

The model explained $41 \%$ of the variability (Nagelkerke Pseudo-R2 $=0.41$ ), with no evidence of poor fit (model $\chi_{38}^{2}=1061.70, p<0.001$; Pearson $\chi_{4720}^{2}=4737.23, p=0.43$, deviance $\left.\chi_{4720}^{2}=4161.02, p=1.00\right)$. For Resolute Cyclists, the odds of belonging to this segment were lower among the UK, Spain, Hungary and Italy compared to Sweden, with small to large effect sizes whereas Dutch cyclists showed a higher probability of belonging to this segment, with a medium effect size. In particular, cyclists from Sweden were three times as likely as those from the U.K. (1/0.28), one and a half times as likely as those from Hungary (1/0.59), six times as likely as those from Italy (1/0.15), and sixteen times as likely as those from Spain (1/0.06) to be in this segment. Dutch cyclists were three times 


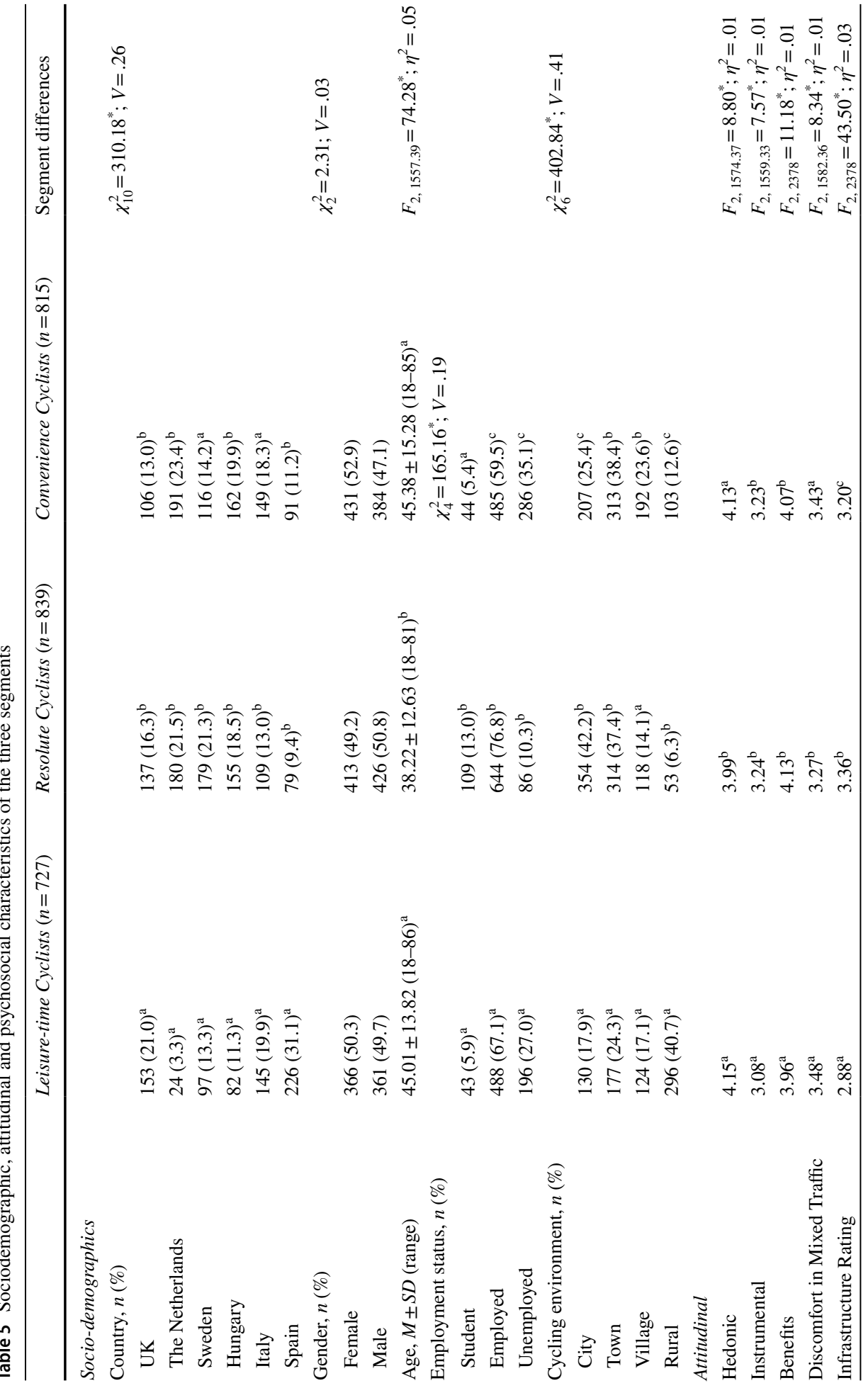




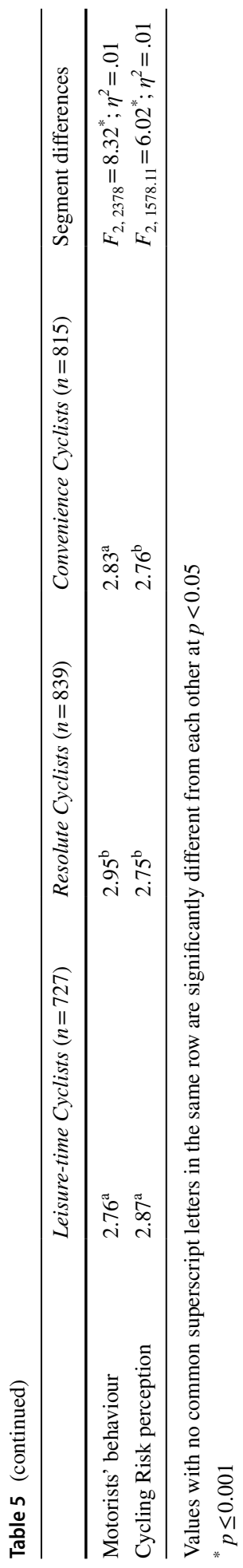


Table 6 Multinomial Logistic Regression of segments on sociodemographic, attitudinal and psychosocial variables

\begin{tabular}{|c|c|c|c|c|}
\hline \multirow[b]{2}{*}{ Independent variable } & \multicolumn{2}{|l|}{ Resolute Cyclists } & \multicolumn{2}{|c|}{ Convenience Cyclists } \\
\hline & B (S.E.) & OR $(95 \% \mathrm{CI})$ & B (S.E.) & OR $(95 \% \mathrm{CI})$ \\
\hline \multicolumn{5}{|l|}{ Socio-demographics } \\
\hline \multicolumn{5}{|l|}{ Country $(\text { Sweden })^{\mathrm{a}}$} \\
\hline UK & $-1.27(0.22)^{* *}$ & $0.28(0.19-0.43)$ & $-0.73(0.21)^{*}$ & $0.48(0.32-0.72)$ \\
\hline The Netherlands & $1.23(0.29)^{* *}$ & $3.42(1.96-5.98)$ & $1.75(0.28)^{* *}$ & $5.75(3.35-9.87)$ \\
\hline Spain & $-2.75(0.23)^{* *}$ & $0.06(0.04-0.10)$ & $-1.71(0.22)^{* *}$ & $0.18(0.12-0.28)$ \\
\hline Hungary & $-0.52(0.23)^{*}$ & $0.59(0.38-0.93)$ & $0.12(0.22)$ & $1.12(0.73-1.73)$ \\
\hline Italy & $-1.89(0.23) * *$ & $0.15(0.10-0.24)$ & $-0.59(0.21)^{*}$ & $0.56(0.37-0.85)$ \\
\hline \multicolumn{5}{|l|}{ Gender $(\text { Female })^{\mathrm{a}}$} \\
\hline Male & $0.06(0.13)$ & $1.06(0.83-1.36)$ & $-0.11(0.12)$ & $0.90(0.71-1.14)$ \\
\hline Age & $-0.02(0.01)^{* *}$ & $0.98(0.97-0.99)$ & $-0.01(0.01)$ & $1.00(0.99-1.01)$ \\
\hline \multicolumn{5}{|l|}{ Job status (Unemployed) ${ }^{\mathrm{a}}$} \\
\hline Employed & $0.98(0.18)^{* *}$ & $2.67(1.88-3.78)$ & $-0.32(0.14)^{*}$ & $0.73(0.55-0.97)$ \\
\hline Students & $0.99(0.30)^{* *}$ & $2.69(1.49-4.86)$ & $-0.45(0.29)$ & $0.64(0.36-1.13)$ \\
\hline \multicolumn{5}{|l|}{ Cycling environment (Rural) ${ }^{\mathrm{a}}$} \\
\hline City & $2.68(0.21)^{* *}$ & $14.62(9.66-22.15)$ & $1.53(0.18)^{* *}$ & $4.61(3.23-6.57)$ \\
\hline Town & $2.14(0.20)^{* *}$ & $8.51(5.74-12.62)$ & $1.56(0.17)^{* *}$ & $4.78(3.46-6.60)$ \\
\hline Village & $1.39(0.23)^{* *}$ & $4.02(2.58-6.26)$ & $1.32(0.19)^{* *}$ & $3.73(2.59-5.38)$ \\
\hline \multicolumn{5}{|l|}{ Attitudinal } \\
\hline Hedonic & $-0.77(0.11)^{* *}$ & $0.46(0.37-0.58)$ & $-0.34(0.11)^{* *}$ & $0.71(0.58-0.88)$ \\
\hline Instrumental & $0.29(0.09)^{* *}$ & $1.34(1.12-1.61)$ & $0.28(0.09)^{*}$ & $1.32(1.11-1.57)$ \\
\hline Benefits & $1.05(0.13)^{* *}$ & $2.87(2.22-3.71)$ & $0.57(0.12)^{* *}$ & $1.77(1.39-2.26)$ \\
\hline Discomfort in Mixed Traffic & $-0.14(0.07)^{*}$ & $0.87(0.76-0.99)$ & $-0.10(0.06)$ & $0.90(0.80-1.02)$ \\
\hline Infrastructure Rating & $0.07(0.07)$ & $1.07(0.94-1.23)$ & $0.10(0.06)$ & $1.10(0.97-1.25)$ \\
\hline Motorists' behaviour & $-0.03(0.08)$ & $0.97(0.83-1.13)$ & $-0.06(0.07)$ & $0.94(0.82-1.08)$ \\
\hline Cycling Risk perception & $-0.04(0.09)$ & $0.96(0.81-1.13)$ & $-0.13(0.08)$ & $0.88(0.75-1.03)$ \\
\hline
\end{tabular}

SE standard error, $C I$ confidence intervals

${ }^{\text {a }}$ Reference category

$* p<0.05$

$* * p \leq 0.001$

more likely to be part of this group compared to Swedish cyclists. Gender was unrelated to being a Resolute cyclist while age and employment status were related to belonging to this group, with small effect sizes. Specifically, young people seemed slightly more likely to be part of the Resolute Cyclists' category since the likelihood of being a member of this segment decreased by $2 \%$ per each additional year of age. Both student and employed cyclists were twice as likely as unemployed cyclists to be members of this segment. Cycling environment was significantly related to belonging to this group, with mediumto-large effect size. Specifically, cyclists making the majority of their cycle trips within a city, town or village environment were, respectively fourteen, eight, and four times more likely than those cycling in a rural environment to be Resolute Cyclists. As to attitudes towards cycling, cyclists with lower hedonic and higher instrumental and benefit-oriented attitudes were more likely to be members of this segment, with small-to-moderate effect 
sizes. Specifically, a one-unit increase in hedonic attitudes was related to a $54 \%$ decrease in the odds of belonging to this segment, while a one-unit increase in instrumental and environment-oriented attitudes was linked, respectively, to a $34 \%$ and a $187 \%$ increase in the odds of being a Resolute Cyclist. Finally, cyclists with lower discomfort while cycling in mixed traffic were slightly more likely to be members of this group. Specifically, a oneunit increase in discomfort in mixed traffic was related to a $13 \%$ decrease in the odds of being a Resolute Cyclist. Cycling infrastructure rating, cycling risk perception and cyclists' evaluation of motorists' behaviour were not related to being Resolute Cyclists.

For Convenience Cyclists, the odds of being a member of this segment were higher among The Netherlands and lowered among the U.K., Spain and Italy compared to Sweden, with small to medium effect sizes. Dutch cyclists were five times more likely to be part of this group. On the contrary, cyclists from Sweden were twice as likely as those from the U.K. (1/0.48) and from Italy (1/0.56) to be Convenience Cyclists and five times as likely from Spain (1/0.19). Gender and age were unrelated to being a Convenience Cyclist, whereas employed cyclists were slightly less likely than unemployed cyclists to be members of this segment. Cycling environment was related to belonging to this group, with medium effect size. Specifically, cyclists cycling in a city or town environment were almost five times, and those cycling in a village environment were almost four times as likely as those cycling in a rural environment to be Convenience Cyclists. As to attitudes towards cycling, lower hedonic and higher instrumental and benefit-oriented attitudes were significantly associated with being a Convenience Cyclist, with small effect sizes. A oneunit increase in hedonic attitudes was related to a $29 \%$ decrease in the odds of belonging to this segment. In contrast, a one-unit increase in instrumental and environment-oriented attitudes was related, respectively, to a $32 \%$ and a $77 \%$ increase in the odds of being a Convenience Cyclist. Discomfort while cycling in mixed traffic, cycling infrastructure rating, cycling risk perception and cyclists' evaluation of motorists' behaviour were not related to being a Convenience Cyclist.

\section{Discussion}

The present study contributed to describing common patterns, attitudes and psychosocial characteristics of segments of cyclists in Europe, which according to other authors is one of the less investigated aspects if compared to other modes of transport (Handy et al. 2014). It was possible to identify three clusters that substantially differ according to cycling trip purpose, modal split and cycling frequency. We want to emphasise that labelling participants according to a transport mode (i.e., cyclists) is, in most cases, simplistic. A more appropriate approach in psychosocial studies would be to use labels that address human behaviour's nuances and complexities. This study aimed to address participants' cycling-related behaviours; thus, we believe that labelling clusters according to the different cycling use patterns would help compare results with other studies and ease the reader.

The first cluster, Leisure-Time cyclists, is composed of people using the bicycle exclusively for sport or recreational activity, mostly in rural areas, with low cycling frequency and a clear preference for using the car for their daily travel. Living in Sweden decrease the probability of belonging to this cluster, especially if compared to the U.K., Spain and Italy. Results suggest that cyclists belonging to this cluster perceive cycling as a pleasant and relaxing activity, and they cycle mainly in rural areas for sport or leisure activities. However, they do not perceive it as a convenient mode of transport nor are interested in the 
benefits that everyday cycling could bring for themselves and society. Age and income play a role. Like older adults, unemployed people are more likely to be Leisure-time Cyclists.

The second cluster, Resolute Cyclists, is composed of cyclists that prefer using the bicycle instead of the private car and cycle often for their everyday trips, especially for commuting. Living in The Netherlands, Hungary or Sweden increases the chance of belonging to this cluster, as well as being younger, student or being employed, which increase the probability of belonging to this cluster. Cyclists in this cluster give importance to the several benefits of cycling while they tend not to perceive it as a pleasant and relaxing activity. Resolute Cyclists are well aware of the benefits of cycling as a healthy and flexible means of transport. Older cyclists are less likely to be Resolute Cyclists; a possible explanation is that they may have health-related issues or reduced mobility. This result is only partially in line with results from Dill and Voros (2007) which showed that younger adults were more likely to be regular cyclists and that the significant drop-off in regular cycling occurred at age 55 years and above. In addition, recent studies have shown that the younger generation (Delbosc and Currie 2013; Kuhnimhof et al. 2012) are more willing to use other modes of transport than the car. As stated by Prillwitz and Bar (2011), younger generations are often defined as "green travellers", suggesting a mode preference for active means of transport, possibly dictated by income constraints or delays in adult life transition (Delbosc and Currie 2014), shifts in attitudes (Vij et al. 2017), differences in their daily activities or even by changes in the local transport or land-use systems (Delbosc et al. 2019). Our analysis highlighted that respondents with higher instrumental and benefit-oriented attitudes were more likely to be members of the Resolute-cyclists segment. This is in line with results from Dill and Voros (2007), which found that respondents with positive attitudes towards cycling were more likely to be regular cyclists.

Higher ratings of discomfort while cycling in mixed traffic decreased the odds of belonging to the Resolute Cyclists cluster. These cyclists are in fact, more likely to cycle in city or town environments where mixed traffic situations are frequent. Sharing the road with motorised vehicles can influence cycling patterns and bicycle usage exhibiting avoidance behaviours. While it would be hasty to infer a causal relationship, in this case, it is still important to acknowledge that to increase cycling levels and prompt citizens to increase bicycle use in urban areas it is essential to address negative feelings arising in the interaction with motorised vehicles' drivers. A study from Chataway et al. (2014) showed that cyclists in an emerging cycling city tend to be more concerned regarding the interaction with motorised vehicles than those in an established cycling city. Our findings are partially in line with our expectations. Even if, contrarily to our expectations, cyclists from the U.K. tend to report higher evaluations of motorists' behaviuour, results show that cyclists from Italy and Spain (which can be considered emerging cycling countries) tend to report lower evaluations of motorists behaviour. This is particularly relevant, as it has been argued that to cope with negative feelings arising from the exposure to motorists' behaviour, cyclists tend to avoid cycling in mixed traffic conditions, and sometimes avoid cycling in general (Prati et al. 2020; Puchades et al. 2018; O'Connor and Brown 2010). This implies that, in low cycling culture countries, promoting cycling becomes particularly difficult if motorised vehicles behaviour is not addressed. In countries such as Italy and Spain, national effort should be targeted at improving interactions between traffic participants. Future studies should address this relationship aiming to investigate to what extent behaviours of traffic participants could actually influence sustainable mobility choices.

The third cluster, Convenience Cyclists, is composed of cyclists that use the bicycle for personal business or recreational activities, have moderate riding frequency and choose the bike in the daily trips similarly to cars. Convenience cyclists mainly cycle in urban 
areas (city and town). The results suggest that participants in this cluster are more likely to live in a strong cycling culture country (i.e., The Netherlands, Hungary) and give importance to the several benefits that cycling holds, in particular environmental-related benefits. At the same time, they tend not to perceive cycling as a pleasant and relaxing activity. Similarly, Dill and Voros (2007) found a significant relationship between environmental values and utilitarian cycling. Respondents who thought air quality was a problem try to limit their driving to help improve air quality, and those who believed that the region did not need to build more highways were more likely to be utilitarian cyclists. Discomfort while cycling in mixed traffic seems not to influence belonging to the Convenience Cyclists cluster. This is an interesting result and suggests that Convenience Cyclists tend to cycle because of necessity or simply out of convenience, regardless feelings of fear or discomfort towards sharing the road with motorised vehicles. This adds to results mentioned in the previous paragraph, stressing that, in urban areas, is also essential to make cycling more advantageous in comparison to other motorised mode of transport. Infrastructural solutions addressing this issue are, for instance, green waves, preferential crossings or "all green" crossings for cyclists at busy intersections.

In line with our findings, an aspect worth discussing is the country of residence and its related societal and infrastructural aspects. According to the Eurobarometer 422 survey (European Commission 2014), the countries studied in the present work differ significantly in terms of the use of the bicycle as the primary means of transport. Present results suggest that in countries where cycling is a well-established means of transport (i.e. The Netherlands, Sweden and Hungary), there is a lower probability of people cycling for leisure as well as a higher chance of belonging to the category of Resolute Cyclists than in countries where the percentage of people who reported cycling on a daily basis is low (i.e. Italy, Spain and the United Kingdom). This could reflect national cycling policies and mobility culture. There are studies in literature exploring the role played by national policies and investments in making cycling safe and popular (Hull and O'Holleran 2014; Kosztin et al. 2017) as well as by the level of infrastructure provided to make cycling attractive (Pucher and Buehler 2008). For example, The Netherlands has been at the forefront of policies to make cycling safe and attractive and, as such, can be considered an exemplary "strong cycling culture" (Hull and O'Holleran 2014). The same reasoning can be applied to Sweden and Hungary, where in recent years efforts have been made to increase the share of cycling in its modal split (Bastian and Börjesson 2018; Koglin 2017; Haustein and Nielsen 2016), a strategy which in turn has been seen to encourage the adoption of this means of transport (Pucher and Buehler 2008).

When discussing infrastructural and societal aspects, it is valuable to mention the concept of human infrastructure as defined by Lugo (2013). Infrastructures are essential to promote bicycle use but also identities and behaviour of traffic participants. It is thus vital to consider the close interplay between the built and social environment. Lugo (2013) argued that social networks, identities and cultural practices could act as human infrastructure, upholding bicycle use growth. For instance, bicyclists' preferred routes are influenced not just by infrastructure, but also by attitudes and participation in particular social networks. Human infrastructure can influence cycling behaviour, both encouraging or discouraging it. According to Lugo (2013) exchange of specialised pieces of information (e.g. when cyclists give each other suggestions on preferred routes based on personal knowledge and not only on municipal maps) or the enactment of an expectation (e.g. aggressive behaviour of motorists) can constitute human infrastructure. Our results somewhat suggest that human infrastructure and cycling patterns are related. For instance, the cluster with higher cycling levels, Resolute Cyclists, also reports the highest ratings of motorists' behaviour 
and higher ratings of provision and infrastructure quality. The study's design does not allow making strong causal inferences, but it is reasonable to expect that people who experience aggressive behaviours from motorists could be discouraged to cycle and ultimately use the bicycle in fewer occasions.

On the notion of human infrastructure, it is relevant to cite a study by Nello-Deakin and Nikolaeva (2020), which identified seven main factors encouraging foreigner newcomers to cycle in Amsterdam. Authors highlighted the critical role of cyclists themselves in encouraging others to cycle. Specifically, echoing a study by Larsen (2017), authors suggested that it is useful to consider cyclists as a form of human infrastructure that plays a crucial role in reproducing the city's cycling culture and fostering cycling uptake by other newcomers. Cycling practices are partly influenced by environmental factors and mostly by interactions between different time and space factors and how these are interlinked into people's lives (Nello-Deakin and Nikolaeva 2020). Cycling cultures can thus be considered intricate systems dependent on a series of causal feedback loops related to social, political, and spatial processes (Macmillan and Woodcock 2017). Simultaneously, traffic participants' human infrastructure also plays an important role in influencing policymakers on the topic of urban mobility, thereby contributing to shaping streets, laws and behaviour (Nello-Deakin and Nikolaeva 2020). As suggested by other authors (e.g. Lugo 2013), we argue that bringing together different cyclists is of utmost importance for cycling advocacy and planning. The present study contributes to highlighting which type of cyclists can be expected in European countries and can guide practitioners and policymakers in involving even bicycle users with lower cycling levels or who could not self-identify as cyclists (e.g. Leisure-time Cyclists or Convenience Cyclists). Their use of street space should also be considered part of the human infrastructure, thus influencing bicycle culture and ultimately use. This is equally useful for countries with strong (e.g. the Netherlands) and emerging cycling cultures (e.g. U.K). Emerging cycling culture countries should leverage on human infrastructure and networks of Resolute Cyclists to ultimately increase population cycling levels. In the study by Nello-Deakin and Nikolaeva (2020), it has been shown that people who already decided to adopt cycling plays a crucial role in fostering cycling uptake in others.

Results regarding cycling frequency imply possible consideration about participant' level of physical activity. Active transport contributes to alleviating the adverse health effects of inactivity and cycling can help reach the WHO's physical activity recommendation, bringing beneficial effects for people's health (Raser et al. 2018). While acquiring precise data on time spent cycling and travelled distance was out of the scope of the present study, an estimation of the days spent cycling per year can provide a rough picture of the amount of physical activity carried out. Resolute Cyclists are the ones achieving the higher level of physical activity with, on average, 173 days spent cycling per year and a preference for using the bicycle instead of the car. Several studies revealed that reducing car usage for the active mode of transport provides benefits to people's health as it counteracts sedentary lifestyles (e. g. De Geus et al. 2008; Basset et al. 2008; Celis-Morales et al. 2017;). Thus, efforts should be focused on Leisure-Time Cyclists, who prefer using the car instead of bicycle and cycle only 56 days per year on average. Convenience Cyclists holds the potential for switching towards daily active mobility since they have no clear preference between the use of the car or the bicycle and cycle around 107 days per year.

Our results suggest that each type of cyclists could be differently responsive to campaign leveraging on different messages. Campaign and intervention targeting Leisure-Time Cyclists could leverage on the hedonic attitudes held by such a group and prompt them to use the bicycle for purposes other than recreation or sport, for instance, messages stressing 
the pleasure of cycling not only as a leisure activity but as an everyday means of transport. It would be advisable to change their bicycle patterns fostering some kind of halo effect (Nisbett and Wilson 1977) since they already report high scores on hedonic attitudes. Leisure-Time Cyclists could be influenced by messages stressing the contextual opportunities and the positive consequences of the cycling mode. Care must be taken though when choosing environmental concerns as core messages in campaign and interventions as other findings claim that information about the adverse environmental effects of the car raises public awareness but is usually insufficient to change behaviour (Tertoolen et al. 1998). In any case, it is essential to assume bidirectional effects between cycling behaviour and its determinants, such as attitudes, preferences, and habits (Handy et al. 2014). Travel behaviour studies established that bidirectional effects exist between attitudes and behaviour (Dobson et al. 1978; Golob et al. 1979; Tardiff 1977).

Moreover, results of the present study can be useful to hone the European Cycling Strategy (ECF, 2017) and provide insights for a future version of the document, specifically regarding policy and behavioural change. Market segmentation has, in fact, proven to be beneficial for tailoring communication and intervention for a specific purpose which is in this case, making cycling safer and more attractive for European Citizens. It is as well useful for directing funds to targeted objectives, maximising the expected impact of interventions and making expenses more efficient. Anable (2005) remarked that the clustering approach illustrates that policy interventions need to be responsive to the different motivations and constraints of the sub-groups. However, such responses may be less about 'harder' infrastructural changes and more about 'softer' interventions with an emphasis on management and marketing activities. As it has been argued by Handy et al. (2014) policymakers can benefit from guidance on which of the possible strategies are likely to increase cycling and to what degree. Further studies can help to identify critical factors associated with cyclist preferences, attitudes and behaviours, pointing to a potentially effective strategy.

The current study has some limitations which should be recognised. First, its cross-sectional nature limits our possibility to make strong causal inferences about present results. In addition, social desirability and recall bias may have affected the results. The study population is self-selective (i.e. online panel) and, therefore, the generalizability of the findings is limited. Central tendency bias, which inclines participants to avoid the endpoints of a response scale and to prefer responses closer to the midpoint (Stevens 1971), might have occurred due to the usage of five-point Likert type scales, which is the kind of scale particularly giving rise to such bias (Douven 2018). Furthermore, respondents' perceptions (e.g. regarding risks, evaluation of motorists' behaviour and infrastructure) are most likely not consistent across countries. It is reasonable to assume that assessments of safety and other factors are strictly dependent on each country's dominant culture and specificities. However, to allow comparisons between clusters and countries, responses are treated equally. Although the present research considers the environment in which participants mainly cycle in, there are many relevant variables related to the built environment that have not been investigated here (e.g., land use mix, population density, where the participants reside). Finally, the present study focuses on cyclists only and lacks insight into other modes of transport (e.g. public transport) and non-cyclists. Regarding future development of the present study, it is worth considering that comparing current results with data from non-cyclists would allow developing better guidance for policymakers. Future research should aim at filling these gaps.

The present study identified clusters of cyclists based on cycling motivation and patterns at a European level and profiled the membership to each cluster according to 
socio-demographics and psychosocial variables. The value of the present study is to highlight commonalities in patterns, characteristics and attitudes of cyclists in six different European countries and it could be useful for policymakers, urban planners and transport experts for developing targeted and tailored measures to increase cycling levels. Our study supports that cycling patterns and habits are linked to culture as well as attitudes and evaluation of the cycling environment, highlighting the importance of the feeling of discomfort in mixed traffic and the area in which people mainly cycle in.

Authors' contributions Federico Fraboni: conceptualisation, methodology, formal analysis, investigation, data curation, writing — original draft, writing - review \& editing. Gabriele Prati: conceptualisation, methodology, writing — review \& editing. Giulia Casu: methodology, formal analysis, writing — original draft. Marco de Angelis: methodology, formal analysis, resources, writing-review \& editing. Luca Pietrantoni: conceptualisation, writing - review \& editing, supervision, project administration, funding acquisition.

Funding Open access funding provided by Alma Mater Studiorum - Università di Bologna within the CRUI-CARE Agreement. This work was supported by the European Commission under the Horizon 2020 Framework Programme (2014-2020). Project XCYCLE (contract number: 635975).

Availability of data and material Not applicable.

Code availability Not applicable.

\section{Compliance with ethical standards}

Competing interest The authors declare they have no actual or potential competing financial interests.

Open Access This article is licensed under a Creative Commons Attribution 4.0 International License, which permits use, sharing, adaptation, distribution and reproduction in any medium or format, as long as you give appropriate credit to the original author(s) and the source, provide a link to the Creative Commons licence, and indicate if changes were made. The images or other third party material in this article are included in the article's Creative Commons licence, unless indicated otherwise in a credit line to the material. If material is not included in the article's Creative Commons licence and your intended use is not permitted by statutory regulation or exceeds the permitted use, you will need to obtain permission directly from the copyright holder. To view a copy of this licence, visit http://creativecommons.org/licenses/by/4.0/.

\section{References}

Ahmed, F., Rose, G., Jakob, C., Hoque, M.R.: Examination of clusters for better understanding commuter cyclists' travel behavior: insights from a longitudinal panel survey. Transp. Res. Rec. 2665(1), 40-50 (2017)

Aldred, R., Crosweller, S.: Investigating the rates and impacts of near misses and related incidents among UK cyclists. J. Transport Health 2(3), 379-393 (2015). https://doi.org/10.1016/j.jth.2015.05.006

Aldred, R., Jungnickel, K.: Why culture matters for transport policy: the case of cycling in the U.K. J. Transp. Geogr. 34, 78-87 (2014)

Anable, J.: 'Complacent car addicts' or 'aspiring environmentalists'? Identifying travel behaviour segments using attitude theory. Transp. Policy 12(1), 65-78 (2005)

Anable, J., Gatersleben, B.: All work and no play? The role of instrumental and affective factors in work and leisure journeys by different travel modes. Transp. Res. Part A Policy Pract. 39(2-3), 163-181 (2005)

Bagley, M.N., Mokhtarian, P.L.: The impact of residential neighborhood type on travel behavior: A structural equations modeling approach. Ann. Reg. Sci. 36(2), 279-297 (2002)

Bamberg, S., Schmidt, P.: Theory-driven evaluation of an intervention to reduce the private car-use. J. Appl. Soc. Psychol. 31(6), 1300-1329 (2001) 
Barberan, A., Monzon, A.: How did bicycle share increase in Vitoria-Gasteiz? Transp. Res. Procedia 18, 312-319 (2016)

Basford, L., Reid, S., Lester, T., Thomson, J., and Tolmie, A.: Drivers' perceptions of cyclists. TRL Report TRL549, Charging and Local Transport Division, Department for Transport. Retrieved from https:// trl.co.uk/sites/default/files,TRL549.pdf (2002).

Bassett, D.R., Pucher, J., Jr., Buehler, R., Thompson, D.L., Crouter, S.E.: Walking, cycling, and obesity rates in Europe, North America, and Australia. J. Phys. Act. Health 5(6), 795-814 (2008)

Bastian, A., Börjesson, M.: The city as a driver of new mobility patterns, cycling and gender equality: Travel behaviour trends in Stockholm 1985-2015. Travel Behav. Soc. 13, 71-87 (2018)

Bauman, A.E., Rissel, C., Garrard, J., Ker, I., Speidel, R., Fishman, E.: Cycling: Getting Australia Moving: Barriers, Facilitators and Interventions to Get More Australians Physically Active Through Cycling. Cycling Promotion Fund, Melbourne (2008)

Bergström, A., Magnusson, R.: Potential of transferring car trips to bicycle during winter. Transp. Res. Part A. Policy Pract. 37(8), 649-666 (2003)

Bill, E., Rowe, D., and Ferguson, N.: Does experience affect perceived risk of cycling hazards?. In: Scottish Transport Applications and Research (STAR) Conference, $20^{\text {th }}$ May, Glasgow, UK (2015)

Burbidge, S., Goulias, K.: Active travel behavior. Transp. Lett. 1(2), 147-167 (2009)

Celis-Morales, C.A., Lyall, D.M., Welsh, P., Anderson, J., Steell, L., Guo, Y., Gill, J.M.: Association between active commuting and incident cardiovascular disease, cancer, and mortality: prospective cohort study. BMJ 357, j1456 (2017)

Chaloux, N., El-Geneidy, A.: Rules of the road: compliance and defiance among the different types of cyclists. Transp. Res. Record (2019). https://doi.org/10.1177/0361198119844965

Chataway, E.S., Kaplan, S., Nielsen, T.A.S., Prato, C.G.: Safety perceptions and reported behavior related to cycling in mixed traffic: a comparison between Brisbane and Copenhagen. Transp. Res. Part F Traffic Psychol. Behav. 23, 32-43 (2014)

Cohen, J.: Statistical power analysis for the behavioral sciences, 2nd edn. Lawrence Erlbaum, Mahwah, NJ (1988)

Damant-Sirois, G., Grimsrud, M., El-Geneidy, A.M.: What's your type: a multidimensional cyclist typology. Transportation 41(6), 1153-1169 (2014)

De Geus, B., Van Hoof, E., Aerts, I., Meeusen, R.: Cycling to work: influence on indexes of health in untrained men and women in Flanders Coronary heart disease and quality of life. Scand. J. Med. Sci. Sports 18(4), 498-510 (2008)

de Nazelle, A., Nieuwenhuijsen, M.J., Anto, J.M., Brauer, M., Briggs, D., Braun-Fahrlander, C., Lebret, E.: Improving health through policies that promote active travel: a review of evidence to support integrated health impact assessment. Environ. Int. 37(4), 766-777 (2011). https://doi.org/10.1016/j. envint.2011.02.003

Delbosc, A., Currie, G.: Causes of youth licensing decline: a synthesis of evidence. Transport Rev. 33(3), 271-290 (2013). https://doi.org/10.1080/01441647.2013.801929

Delbosc, A., Currie, G.: Impact of attitudes and life stage on decline in rates of driver's license acquisition by young people in Melbourne Australia. Transp. Res.ch Record 2452(1), 62-70 (2014). https://doi. org/10.3141/2452-08

Delbosc, A., McDonald, N., Stokes, G., Lucas, K., Circella, G., Lee, Y.: Millennials in cities: Comparing travel behaviour trends across six case study regions. Cities 90, 1-14 (2019). https://doi.org/10. 1016/j.cities.2019.01.023

Dill, J., Voros, K.: Factors affecting bicycling demand: initial survey findings from the Portland, Oregon, region. Transp. Res. Rec. 2031(1), 9-17 (2007)

Dobson, R., Dunbar, F., Smith, C.J., Reibstein, D., Lovelock, C.: Structural models for the analysis of traveler attitude-behavior relationships. Transportation 7(4), 351-363 (1978). https://doi.org/10.1007/ BF00168036

Douven, I.: A Bayesian perspective on Likert scales and central tendency. Psychon. Bull. Rev. 25(3), 12031211 (2018)

European Commission (2014). Special Eurobarometer 422a “Quality of Transport”. December, 2014. Retrieved from http://ec.europa.eu/public_opinion/archives/ebs/ebs_422a_en.pdf

European Cyclists' Federation, ECF. (2017). E.U. Cycling Strategy. Recommendation for Delivering Green Growth and an Effective Mobility System in 2030. Bruxelles. Retrieved from https://ecf.com/sites/ ecf.com/files/EUCS_full_doc_small_file.pdf

Félix, R., Moura, F., Clifton, K.J.: Typologies of urban cyclists: review of market segmentation methods for planning practice. Transp. Res. Rec. 2662(1), 125-133 (2017)

Félix, R., Moura, F., Clifton, K.J.: Maturing urban cycling: Comparing barriers and motivators to bicycle of cyclists and non-cyclists in Lisbon Portugal. J. Transport Health 15, 100628 (2019) 
Fernández-Heredia, Á., Jara-Díaz, S., Monzón, A.: Modelling bicycle use intention: the role of perceptions. Transportation 43(1), 1-23 (2016)

Friedman, H.S.: The Oxford handbook of health psychology. Oxford University Press (2011)

Fruhen, L.S., Flin, R.: Car driver attitudes, perceptions of social norms and aggressive driving behaviour towards cyclists. Accid. Anal. Prev. 83, 162-170 (2015). https://doi.org/10.1016/j.aap.2015.07.003

Garrard, J., Handy, S., Dill, J.: Women and cycling. In: Pucher, J.R., Buehler, R. (eds.) City Cycling, vol. 2012, pp. 211-234. MIT Press, Cambridge, MA (2012)

Gatersleben, B., Appleton, K.M.: Contemplating cycling to work: Attitudes and perceptions in different stages of change. Transp. Res. Part A Policy Pract. 41(4), 302-312 (2007)

Gatersleben, B., Uzzell, D.: Affective appraisals of the daily commute: Comparing perceptions of drivers, cyclists, walkers, and users of public transport. Environ. Behav. 39(3), 416-431 (2007)

Golob, T.F., Horowitz, A.D., Wachs, M.: Attitude-behaviour relationships in travel-demand modelling. In: Hensher, D.A., Stopher, P.R. (eds.) Behavioural travel modelling, pp. 739-757. Croom-Helm, London (1979)

Götschi, T., Garrard, J., Giles-Corti, B.: Cycling as a part of daily life: a review of health perspectives. Transport Rev. 36(1), 45-71 (2016). https://doi.org/10.1080/01441647.2015.1057877

Gulian, E., Glendon, A.I., Matthews, G., Davies, D.R., Debney, L.M.: The stress of driving: a diary study. Work Stress 4(1), 7-16 (1990)

Handy, S., Cao, X., Mokhtarian, P.: Correlation or causality between the built environment and travel behavior? Evidence from Northern California. Transport Res. Part D: Trans. Environ. 10(6), 427444 (2005)

Handy, S., Van Wee, B., Kroesen, M.: Promoting cycling for transport: research needs and challenges. Transport Rev. 34(1), 4-24 (2014)

Haustein, S.: Mobility behavior of the elderly: an attitude-based segmentation approach for a heterogeneous target group. Transportation 39(6), 1079-1103 (2012)

Haustein, S., Hunecke, M.: Reduced use of environmentally friendly modes of transportation caused by perceived mobility necessities: an extension of the theory of planned behavior. J. Appl. Soc. Psychol. 37(8), 1856-1883 (2007)

Haustein, S., Nielsen, T.A.S.: European mobility cultures: A survey-based cluster analysis across 28 European countries. J. Trans. Geogr. 54, 173-180 (2016). https://doi.org/10.1016/j.jtrangeo.2016. 05.014

Haustein, S., Koglin, T., Nielsen, T.A.S., Svensson, Å.: A comparison of cycling cultures in Stockholm and Copenhagen. Int. J. Sustain. Transp. 14(4), 280-293 (2020)

Heesch, K.C., Sahlqvist, S., Garrard, J.: Gender differences in leisure-time and transport cycling: a cross-sectional mixed-methods comparison of cycling patterns, motivators, and constraints. Int. J. Behav. Nutr. Phys. Act. 9(1), 106 (2012)

Heinen, E., Handy, S.: Similarities in attitudes and norms and the effect on bicycle commuting: Evidence from the bicycle cities Davis and Delft. Int. J. Sustain. Trans. 6(5), 257-281 (2012). https:// doi.org/10.1080/15568318.2011.593695

Heinen, E., Maat, K., Van Wee, B.: Day-to-day choice to commute or not by bicycle. Transp. Res. Rec. 2230(1), 9-18 (2011a)

Heinen, E., Maat, K., Van Wee, B.: The role of attitudes toward characteristics of bicycle commuting on the choice to cycle to work over various distances. Transp. Res. Part D Trans. Environ. 16(2), 102-109 (2011b)

Heinen, E., Van Wee, B., Maat, K.: Commuting by bicycle: an overview of the literature. Transport Rev. 30(1), 59-96 (2010)

Hosmer, D.W., Lemeshow, S.: Applied logistic regression, 2nd edn. Wiley, New York, NY (2000)

Hull, A., O'Holleran, C.: Bicycle infrastructure: can good design encourage cycling? Urban Plan. Transport Res. 2(1), 369-406 (2014). https://doi.org/10.1080/21650020.2014.955210

Jensen, M.: Passion and heart in transport-a sociological analysis on transport behaviour. Transp. Policy 6(1), 19-33 (1999)

Jensen, S. U., Rosenkilde, C., and Jensen, N. Road safety and perceived risk of bicycles facilities in Copenaghen. Research report, Road Park, City of Copenaghen. Retrived from http://www.vehic ularcyclist.com/copenaghen1.pdf (2007).

Johnson, M., Chong, D., Carroll, J., Katz, R., Oxley, J., Charlton, J. L.: Naturalistic cycling study: identifying risk factors for cyclists in the Australian Capital Territory. MUARC (2014)

Karanikola, P., Panagopoulos, T., Tampakis, S., Tsantopoulos, G.: Cycling as a smart and green mode of transport in small touristic cities. Sustainability 10(1), 268 (2018). https://doi.org/10.3390/su100 10268 
Kelly, P., Kahlmeier, S., Gotschi, T., Orsini, N., Richards, J., Roberts, N., Scarborough, P., Foster, C.: Systematic review and meta-analysis of reduction in all-cause mortality from walking and cycling and shape of dose response relationship. Int. J. Behav. Nutr. Phys. Act. 11, 132 (2014). https://doi. org/10.1186/s 12966-014-0132-x

Koglin, T.: Urban mobilities and materialities - a critical reflection of "sustainable" urban development. Appl. Mobilities 2(1), 32-49 (2017). https://doi.org/10.1080/23800127.2017.1285169

Koglin, T., Rye, T.: The marginalisation of bicycling in Modernist urban transport planning. J. Transport Health 1(4), 214-222 (2014). https://doi.org/10.1016/j.jth.2014.09.006

Koslowsky, M.: Commuting stress: problems of definition and variable identification. Appl. Psychol. 46(2), 153-173 (1997)

Kosztin, N., Tőzsér, J., Csernoch, L., and Balatoni, I. Reasons for and obstacles to cycling in opinions of residents of Debrecen, Hungary. APSTRACT Appl. Stud. Agribus. Commer. 11(1033-20182952), 53-60 (2017)

Kroesen, M., Handy, S.: The relation between bicycle commuting and non-work cycling: results from a mobility panel. Transportation 41(3), 507-527 (2014)

Kuhnimhof, T., Buehler, R., Wirtz, M., Kalinowska, D.: Travel trends among young adults in Germany: increasing multimodality and declining car use for men. J. Transp. Geogr. 24, 443-450 (2012). https://doi.org/10.1016/j.jtrangeo.2012.04.018

Larsen, J., El-Geneidy, A.: A travel behavior analysis of urban cycling facilities in Montréal, Canada. Transp. Res. Part D. Trans. Environ. 16(2), 172-177 (2011)

Larsen, J.: The making of a pro-cycling city: social practices and bicycle mobilities. Environ. Plan. A 49(4), 876-892 (2017)

Lawson, A.R., Pakrashi, V., Ghosh, B., Szeto, W.Y.: Perception of safety of cyclists in Dublin City. Accid. Anal. Prev. 50, 499-511 (2013). https://doi.org/10.1016/j.aap.2012.05.029

Lugo, A.E.: CicLAvia and human infrastructure in Los Angeles: ethnographic experiments in equitable bike planning. J. Transp. Geogr. 30, 202-207 (2013)

Macmillan, A., Woodcock, J.: Understanding bicycling in cities using system dynamics modelling. J. Transport Health 7, 269-279 (2017)

Macmillan, A., Connor, J., Witten, K., Kearns, R., Rees, D., Woodward, A.: The societal costs and benefits of commuter bicycling: Simulating the effects of specific policies using system dynamics modeling. Environ. Health Perspect. 122(4), 335-344 (2014). https://doi.org/10.1289/ehp.1307250

Majumdar, B.B., Mitra, S., Pareekh, P.: On identification and prioritisation of motivators and deterrents of bicycling. Transp. Lett. 12(9), 591-603 (2020)

Manton, R., Rau, H., Fahy, F., Sheahan, J., Clifford, E.: Using mental mapping to unpack perceived cycling risk. Accid. Anal. Prev. 88, 138-149 (2016). https://doi.org/10.1016/j.aap.2015.12.017

Martha, C., Delhomme, P.: Risk comparative judgments while driving a car among competitive road cyclists and non-cyclists. Transp. Res. Part F Traffic Psychol. Behav. 12(3), 256-263 (2009)

Mercado, R., Páez, A.: Determinants of distance traveled with a focus on the elderly: a multilevel analysis in the Hamilton CMA Canada. J. Transport Geogr. 17(1), 65-76 (2009)

Moudon, A.V., Lee, C., Cheadle, A.D., Collier, C.W., Johnson, D., Schmid, T.L., Weather, R.D.: Cycling and the built environment, a U.S. perspective. Trans[. Res. Part D Trans. Environ. 10(3), 245-261 (2005)

Mueller, N., Rojas-Rueda, D., Salmon, M., Martínez, D., Ambros, A., Brand, C., de Nazelle, A., Dons, E., Gaupp-Berghausen, M., Gerike, R., Götschi, T., Iacorossi, F., Int Panis, L., Kahlmeier, S., Raser, E., Nieuwenhuijsen, M.: Health impact assessment of cycling network expansions in European cities. Prev. Med. 109, 62-70 (2018)

Muñoz, B., Monzon, A., López, E.: Transition to a cyclable city: Latent variables affecting bicycle commuting. Transp. Res. Part a. Policy Pract. 84, 4-17 (2016)

Nello-Deakin, S., \& Nikolaeva, A.: The human infrastructure of a cycling city: Amsterdam through the eyes of international newcomers. Urban Geography, 1-23, (2020)

Nieuwenhuijsen, M.J., Khreis, H.: Car free cities: Pathway to healthy urban living. Environ. Int. 94, 251262 (2016). https://doi.org/10.1016/j.envint.2016.05.032

Nisbett, R.E., Wilson, T.D.: The halo effect: evidence for unconscious alteration of judgments. J. Pers. Soc. Psychol. 35(4), 250 (1977)

Nkurunziza, A., Zuidgeest, M., Van Maarseveen, M.: Identifying potential cycling market segments in Dares-Salaam Tanzania. Habitat Int. 36(1), 78-84 (2012)

O'Connor, J.P., Brown, T.D.: Riding with the sharks: Serious leisure cyclists' perceptions of sharing the road with motorists. J. Sci. Med. Sport 13(1), 53-58 (2010). https://doi.org/10.1016/j.jsams.2008.11. 003

Oosterhuis, H.: Cycling, modernity and national culture. Soc. Hist. 41(3), 233-248 (2016) 
Parkin, J., Wardman, M., Page, M.: Models of perceived cycling risk and route acceptability. Accid. Anal. Prev. 39, 364-371 (2007). https://doi.org/10.1016/j.aap.2006.08.007

Prati, G.: Gender equality and women's participation in transport cycling. J. Transp. Geogr. 66, 369-375 (2018)

Prati, G., De Angelis, M., Fraboni, F., Pietrantoni, L., Johnson, D., and Shires, J. Journey attributes, e-bike use, and perception of driving behavior of motorists as predictors of bicycle crash involvement and severity. Transp. Res. Record, 0361198120953787 (2020).

Prati, G., Fraboni, F., De Angelis, M., Pietrantoni, L., Johnson, D., Shires, J.: Gender differences in cycling patterns and attitudes towards cycling in a sample of European regular cyclists. J. Transp. Geogr. 78, 1-7 (2019)

Prati, G., Puchades, V.M., Pietrantoni, L.: Cyclists as a minority group? Transp. Res. Part F Traffic Psychol. Behav. 47, 34-41 (2017)

Prillwitz, J., Barr, S.: Moving towards sustainability? Mobility styles, attitudes and individual travel behaviour. J. Transp. Geogr. 19(6), 1590-1600 (2011). https://doi.org/10.1016/j.jtrangeo.2011.06.011

Puchades, V.M., Fassina, F., Fraboni, F., De Angelis, M., Prati, G., de Waard, D., Pietrantoni, L.: The role of perceived competence and risk perception in cycling near misses. Saf. Sci. 105, 167-177 (2018)

Pucher, J., Buehler, R.: Making cycling irresistible: lessons from the Netherlands Denmark and Germany. Transport Rev. 28(4), 495-528 (2008)

Pucher, J., and Buehler, R.: Promoting cycling for daily travel: conclusions and lessons from across the globe. City cycling, 347-63, (2012).

Pucher, J., Buehler, R.: Cycling towards a more sustainable transport future. Transp. Rev. (2017). https:// doi.org/10.1080/01441647.2017.1340234

Pucher, J., Dijkstra, L.: Making walking and cycling safer: Lessons from Europe. Transp. Q. 54(3), 25-50 (2000)

Raser, E., Gaupp-Berghausen, M., Dons, E., Anaya-Boig, E., Avila-Palencia, I., Brand, C., Panis, L.I.: European cyclists' travel behavior: Differences and similarities between seven European (PASTA) cities. J. Trans. Health 9, 244-252 (2018)

Rayaprolu, H.S., Llorca, C., Moeckel, R.: Impact of bicycle highways on commuter mode choice: a scenario analysis. Environ. Plan. Urban Anal. City Sci. (2018). https://doi.org/10.1177/2399808318 797334

Rondinella, G. (2015). Considering cycling for commuting: the role of mode familiarity: an exploration on the (circular) relation between cycling behaviours and attitudes toward cycling in VitoriaGasteiz, Spain Doctoral dissertation, Caminos.

Sarstedt, M., Mooi, E.: A concise guide to market research: The process, data, and methods using IBM SPSS Statistics, 2nd edn. Springer, Heidelberg (2014)

Schepers, P., Hagenzieker, M., Methorst, R., van Wee, B., Wegman, F.: A conceptual framework for road safety and mobility applied to cycling safety. Accid. Anal. Prev. 62, 331-340 (2014). https://doi. org/10.1016/j.aap.2013.03.032

Schwarzer, R.: Self-regulatory processes in the adoption and maintenance of health behaviors. J. Health Psychol. 4(2), 115-127 (1999)

Sener, I.N., Eluru, N., Bhat, C.R.: Who are bicyclists? Why and how much are they bicycling? Transp. Res. Rec. 2134(1), 63-72 (2009)

Seyranian, V., Atuel, H., Crano, W.D.: Dimensions of majority and minority groups. Group Process Intergroup Relat 11(1), 21-37 (2008). https://doi.org/10.1177/1368430207084843

Siman-Tov, M., Jaffe, D.H., Peleg, K., and Israel Trauma Group. Bicycle injuries: a matter of mechanism and age. Accid. Anal. Prev., 44(1), 135-139 (2012)

Song, Y., Preston, J., Ogilvie, D.: New walking and cycling infrastructure and modal shift in the U.K.: a quasi-experimental panel study. Transp. Res. Part A Policy Pract. 95, 320-333 (2017)

Stevens, S.S.: Issues in psychophysical measurement. Psychol. Rev. 78, 426-450 (1971). https://doi.org/ $10.1037 / \mathrm{h} 0031324$

Stinson, M.A., Bhat, C.R.: Frequency of bicycle commuting: internet-based survey analysis. Transp. Res. Rec. 1878(1), 122-130 (2004)

Streit, T., Weiss, C., Chlond, B., and Vortisch, P.: More Cycling Or More Cyclists - Dynamics And Potentials Of Bicycle Use In Germany By A Cluster Based Approach. Paper presented at the $93^{\text {rd }}$ Transportation Research Board Annual Meeting, Washington D.C., USA (2014)

Susilo, Y.O., Maat, K.: The influence of built environment to the trends in commuting journeys in the Netherlands. Transportation 34(5), 589-609 (2007)

Susilo, Y.O., Waygood, E.O.D.: A long-term analysis of the mechanisms underlying children's activitytravel engagements in the Osaka metropolitan area. J. Transp. Geogr. 20(1), 41-50 (2012) 
Susilo, Y. O., Williams, K., Lindsay, M., and Dair, C.: Does Green in Mean Green out? An Exploration of Individual Travel Patterns and the Influence of Their Environmental Preferences in U.K." Sustainable Neighborhoods" (No. 10-2131) (2010)

Tardiff, T.J.: Causal inferences involving transportation attitudes and behavior. Trans. Res. 11(6), 397404 (1977). https://doi.org/10.1016/0041-1647(77)90004-1

Tertoolen, G., Van Kreveld, D., Verstraten, B.: Psychological resistance against attempts to reduce private car use. Transp. Res. Part A Policy Pract. 32(3), 171-181 (1998)

Ul-Abdin, Z., De Winne, P., De Backer, H.: Risk-perception formation considering tangible and nontangible aspects of cycling: a flemish case study. Sustainability 11(22), 6474 (2019)

Useche, S.A., Montoro, L., Sanmartin, J., Alonso, F.: Healthy but risky: a descriptive study on cyclists' encouraging and discouraging factors for using bicycles, habits and safety outcomes. Transp. Res. Part F Traffic Psychol. Behav. 62, 587-598 (2019)

Van Lange, P.A.M., Van Vugt, M., Meertens, R.M., Ruiter, R.: A social dilemma analysis of commuting preferences. The role of social value orientation and trust. J. Appl. Soc. Psychol. 28(9), 796-820 (1998)

Vij, A., Gorripaty, S., Walker, J.L.: From trend spotting to trend' splaining: Understanding modal preference shifts in the San Francisco Bay Area. Transp. Res. Part A Policy Pract. 95, 238-258 (2017). https://doi.org/10.1016/j.tra.2016.11.014

Wegman, F., Zhang, F., Dijkstra, A.: How to make more cycling good for road safety? Accid. Anal. Prev. 44(1), 19-29 (2012). https://doi.org/10.1016/j.aap.2010.11.010

Weinstein, N.D.: Unrealistic optimism about future life events. J. Pers. Soc. Psychol. 39(5), 806 (1980)

Winters, M., Davidson, G., Kao, D., Teschke, K.: Motivators and deterrents of bicycling: comparing influences on decisions to ride. Transportation 38(1), 153-168 (2011)

Wittmann, K., Savan, B., Ledsham, T., Liu, G., Lay, J.: Cycling to high school in Toronto, Ontario, Canada: Exploration of school travel patterns and attitudes by gender. Transp. Res. Rec. 2500(1), 9-16 (2015)

Woodcock, J., Tainio, M., Cheshire, J., O’Brien, O., Goodman, A.: Health effects of the London bicycle sharing system: health impact modelling study. BMJ 348, g425 (2014)

Publisher's Note Springer Nature remains neutral with regard to jurisdictional claims in published maps and institutional affiliations.

Federico Fraboni holds a Ph.D. in Psychology at the University of Bologna and conducted extensive research on psychosocial characteristics related to cycling patterns and predictors of bicycle crashes. $\mathrm{He}$ has a background in Work, Organisational, and Personnel Psychology. He participated in many Europeanfunded research and innovation projects as well as collaborating with private companies in internally funded projects. He is currently a researcher in the Human Factors Risk and Safety research unit of the University of Bologna, focusing on human-technology interaction, human-robot collaboration, and safety in high-risk organizations. His main research interests and areas of intervention are acceptability and users' behavioral adaptation of new technologies, human-robot collaboration in industrial settings, human-machine interfaces, and risky behaviors at work.

Gabriele Prati is currently senior assistant professor (fixed-term) at the Department of Psychology of the University of Bologna. He teaches Theories and Methods of Community Psychology and Social Psychology and Deviance. His research interests focus on applied psychology.

Giulia Casu Psychologist and Ph.D., she is Senior Assistant Professor in Psychometrics and member of the research group P.A.T. (Psychometrics, Assessment, and Testing) at the University of Bologna, Italy. She is an expert in methodology, survey development and data analysis. Her research focuses on the development, intercultural adaptation and validation of self-report measures to assess intra- and inter-personal dimensions related to psychological well-being, and the application of advanced data analysis techniques to psychological issues in the field of quality of life, health psychology, and close relationships. She has participated as a methodological advisor or project statistician in many national and international competitively funded, peerreviewed research projects.

Marco De Angelis, graduated in Work and Organisational Psychology, since 2012 he has been conducting academic teaching and also consultancy activities for professionals, mainly in the healthcare context, providing training on organisational well-being, non-technical skills, work-related stress and mental health, risk and safety management. He obtained his $\mathrm{PhD}$ in Psychology at the University of Bologna, conducting 
research on the promotion of sustainable and active mobility and its psychosocial determinants in a public organisation. His main research interests focus on social and behavioural sciences applied to high-risk contexts such as industrial, organisational and traffic environments.

Luca Pietrantoni is a Full Professor at the University of Bologna. He is the coordinator of the EU-funded project H-WORK and the Director of Second cycle degree in Work, Organisational and Personnel Psychology at the University of Bologna (Italy). His main research interests and areas of intervention include human factors, human-machine interface, interventions on health and safety in organisations. He has different publications in international academic journals. 